\title{
Haematopoietic Recovery On Induction Thèrapy In Acute Lymphoblastic Leukaemia By Automated Reticulocyte Analysis
}

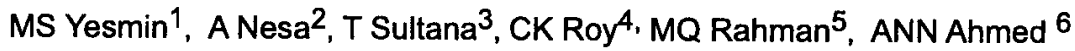

\begin{abstract}
Acute lymphoblastic leukemia (ALL) is the most common childhood malignancy in Bangladesh. ALL is treated with chemotherapy due to high responsiveness. After chemotherapy infection may occur which increases the time of aplasia. For this purpose, reliable laboratory tests that will indicate early haematological recovery are needed. At present, absolute neutrophil count (ANC), reticulocyte counts and peripheral blood film (PBF) examination are used after chemotherapy for prediction of bone marrow recovery. Reticulocyte quantification in the peripheral blood samples, as a percentage or absolute count with immature reticulocyte fraction (IRF) are a reliable measure of haematological recovery established by many studies. This cross sectional study was carried out to evaluate the haematopoietic recovery in children with ALL by automated reticulocyte analysis. Total fifty patients were enrolled in this study on remission induction phase. They received the drugs of the protocol of UKALL-XI. All patients were between 8 months to 15 years age range with a mean age of $5.5 \pm 3.2$. In this study the recovery of reticulocyte percentage occurred at a median of 20 days; ARC 18 days; IRF 16 days; HFR 18 days and ANC was obtained after a median of 23 days. This study established that among the various parameters IRF recovered earlier than others.
\end{abstract}

Keywords: Immature Reticulocyte Fraction(IRF), High fluorescent reticulocyte (HFR), Low fluorescent reticulocyte (LFR), Middle fluorescent reticulocyte (MFR), Absolute neutrophil count (ANC), Absolute Reticulocyte count(ARC).

\section{Introduction}

The childhood leukaemia represents about $35 \%$ of all childhood malignancies and ALL is the commonest form of them ${ }^{1}$. In USA annually 25003500 children are diagnosed with $\mathrm{ALL}^{2}$. After diagnosis of ALL polychemotherapy may be given to the patient on different phases ${ }^{3}$. For early detection of responsiveness or unresponsiveness of chemotherapy monitoring of bone marrow recovery are needed ${ }^{3}$. Among the conventional haematoilogical parameters reticulocyte count with IRF has been establishehd as a reliable indicator in different studies ${ }^{4}$. Reticulocytes are immature red blood cells. They are released in the peripheral blood after a period of maturation in the bone marrow and undergo further differentiation into mature $\mathrm{RBC}^{5}$. In automated flowcytometry method, reticulocyts have been classified morphologically into three maturational stages:low (LFR), middle (MFR) and high fluroscent reticulocytes (HFR) ${ }^{6}$. Immature Reticulocyte Fraction (IRF) is defined as the ratio of young or immature reticulocytes to the total number of reticulocytes. They are larger, having the greatest light scatter properties due to the highest level of RNA. Immature reticulocytes normally constitute less than five percent $(5 \%)$ of the total number of reticulocytes ${ }^{7}$. It is released into the peripheral blood during periods of intense erythropoietic stimulation, An increase in the reticulocyte percent $>1 \%$ or an increase in the absolute reticulocyte count $\geq 50 \times 10^{9} / \mathrm{L}$ is used as an indicator of erythroid regeneration 2,8 . Spanish Multicentric Study Group for Haematopoietic recovery defined IRF > 5\% and HFR > 3\% as recovery ${ }^{8}$. Absolute neutrophil count (ANC) is defined as the number of mature neutrophils plus bands per unit of volume generally accepted as a primary indicator of successful bone marrow recovery. An increase in ANC $\geq 0.5 \times 10^{9} / \mathrm{L}$ defines successful myeloid recovery after chemotherapy $4,9,10$. The aim of this study is to find out sensitive indicator of marrow recovery among the reticulocyte subpopulations assesed by automated method in children with ALL.

\section{Materials and Methods}

This cross sectional study was carried out in the Department of Clinical Pathology and Department of Paediatric Haematology and Oncology, BSMMU, Dhaka during the period of October' 2009 to

\section{Dr. Mst. Shaila Yesmin, Assistant Professor, Dept. of Pathology, International Medical College Hospital.}

2. Dr. Ayatun Nesa. Asstt Professor, Dept. of Pathology, Ibrahim Medical College.

3. Dr. Tuhin sultana. Associate Professor, Department of Clinical Pathology. BSMMU.

4. Dr. Chandan Kumar Roy, Assistant professor, Department of Clinical Pathology, BSMMU.

5. Dr. Md. Quddusur Rahman, Associate Professor, Department of Clinical Pathology, BSMMU.

6. Dr. A. N. Nashimuddin Ahmed, Professor and chairman, Department of Clinical Pathology, BSMMU. 
September' 2010. 50 Children upto 18 years of age irrespective of sex with acute lymphoblastic leukaemia attended in Paediatric Hemato-Oncology outpatients \& inpatients department for treatment included in this study. Reticulocyte counting was carried out in the Sysmex xt-2000i automated haematology analyzer with reticulocyte counter. Blood sample (2 ml) was collected in an EDTA tube for complete blood count (CBC), ANC, reticulocyte profile (both manual and automated method) and PBF examination. Count was done preferably within $2 \mathrm{hrs}$ of collection. If delayed, blood sample was kept at $4^{\circ} \mathrm{C}$ before test. ANC was calculated from total leucocyte count and differential count of WBC.

Supravital staining of unfixed RBCs was done with new methylene blue (NMB) in 50 patients and 40 controls. Then mixing of $100 \mu \mathrm{l}$ whole blood with 100 $\mu \mathrm{l}$ of $1 \%$ NMB solution in a $10 \times 75-\mathrm{mm}$ tube. After incubation at $37^{0}$ for 15-20 minutes, the dilution was remixed and a wedge smear was performed ${ }^{11}$. The number of reticulocytes per $1000 \mathrm{RBCs}$ determined microscopically on $\times 100$ objective. Then percentage of reticulocyte was done. A reticulocyte was defined as a RBC containing at least 2 granules of reticulum(Figure-I). Absolute reticulocyte count was calculated from RBC count obtained from automated haematology analyzer.

Automated reticulocyte count was done in 50 patients in Sysmex xt-2000i haematology analyzer. The measuring principle of the system is based on flow cytometry combined with hydrodynamic focusing. EDTA blood $(100 \mu \mathrm{l})$ is aspirated and intracellular RNA is stained by auramine $O$, which is fluroscent under argon laser light ${ }^{12}$. The reticulocyte population is further subdivided into LFR, MFR and HFR (Figure-II). The percentage of reticulocyte is given as the sum of LFR, MFR and HFR. Approximately $3.2 \times 10^{4} \mathrm{RBC}$ are usually analyzed for each sample. The IRF was manually calculated by MFR plus HFR. IRF $>5 \%$ were taken as bone marrow recovery ${ }^{2}$. Serial hemogram with reticulocyte count, IRF, ANC, and Platelet count measurements was done in every 2-4 days interval upto 32 days of the therapy.

All necessary and relevant data were processed. Data were evaluated by standard statistical methods. Analysis was done by SPSS (Statistical package for social science) by applying appropriate formula. Bone marrow recovery were evaluated by Mean, Median, Unpaired t test, Paired $t$ test, Co-efficient of variance test, ANOVA test.

\section{Results}

In present study randomly enrolled 50 patients were taken. Fiftey children were 8 months to 15 years of age range and mean age of the patients was 5.5 \pm 3.2 years. Maximum patients were male. Male and female ratio was $1.5: 1$. In this study during induction remission phase $\mathrm{Hb}$ level were gradually decreased upto $12^{\text {th }}$ day $(p<0.05)$. However from $16^{\text {th }}$ day onwards the $\mathrm{Hb}$ level remain the similar with baseline status which was not significant $(p>0.05)$. At diagnosis mean total count of RBC was $4.0 \pm 0.9$ $\left(\times 10^{12} / L\right)$. During induction remission total count of RBC was significantly declined upto $20^{\text {th }}$ day $(p<0.05)$. At diagnosis mean total count of WBC was 14.74 $25.47\left(\times 10^{9} / \mathrm{L}\right)$. During induction remission total count of WBC was sharply declined at day 8 and continued up to last follow up. Statistically significant differences were observed between different follow-ups day $(p<0.05)$.

The platelet count was declined significantly $(p<0.05)$ upto $16^{\text {th }}$ days then remain the same with the baseline status. Recovery of platelet counts occurred at a median of 24 day, which was 1 day delayed, to recovery of ANC (Table-I).

In this study reticulocyte (ret) \% significantly declined upto day 4 and then gradually increased or remain the same with the diagnosis day in both automated and manual method. Reticulocyte \% recovery occurred first day of persistent 3-days rise of the reticulocyte (ret) from the 18 th day to 28 days (20 days), which was 4 days later than IRF (Table-II). The absolute reticulocyte concentration significantly declined upto day 4 then it remain the same upto last follow up. It recovered at a median of 18 days 2 days later than IRF(Table-II).

This study showed IRF declined gradually in 24 cases and reaches to low-level mean of $12.1 \pm 7.1 \%$ with a median of 6 days (days 4-8). But IRF showed high range at diagnosis before the chemotherapy. Recovery day was ranged from $16.6 \pm 4.6$ (median day 16)(Table-1).

This study showed the HFR status declined significantly upto day 8 and then increased upto $28^{\text {th }}$ day. The HFR recovery occurred from 10 to 32 days with a median of 18 days(Table-II), which was 2 days later than IRF but 5 days earlier than ANC.

In this study the children were profoundly neutropenic $(<500 / \mathrm{ml})$ in day 12 and day 16 . The ANC were remain the same with the day of diagnosis from day 20 to 32 day of the therapy. The ANC were significantly $(p<0.05)$ declined upto day 16 of therapy. The range of ANC recovery was 10-32 days (median 23 day) (Table-1). 


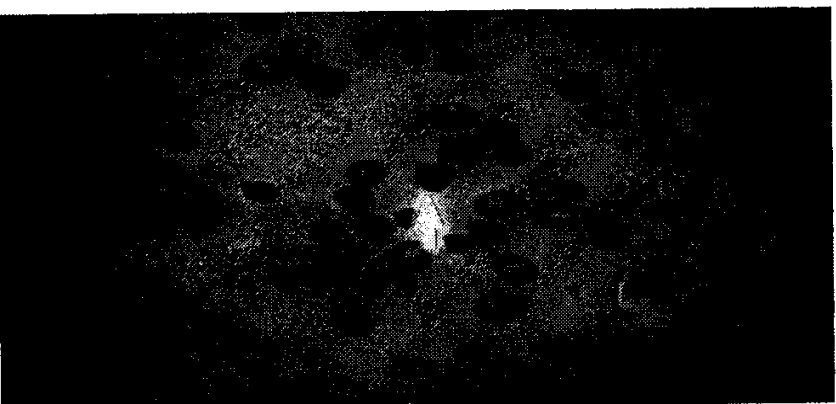

Figure 1 : Photomicrograph of reticulocytes in children with ALL on day 16 induction of remission phase. Stained supravitally by new methylene blue $(\times 1000)$.

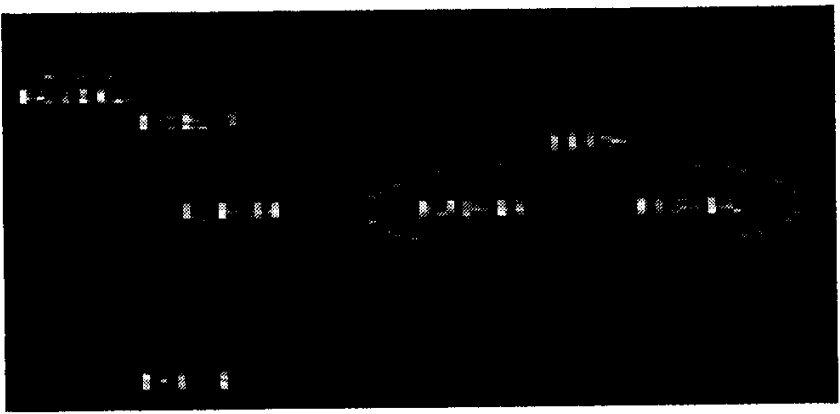

Figure 2 : IRF scattergram in Sysmex XT-2000i hematology analyzer

Table I

Comparison of IRF, ANC and Platelet recovery in days $(n=50)$

Recovery in days $P$ Value

IRF

$\begin{array}{lll}\text { Mean } \pm \text { SD } & 16.6 \pm 4.6 & 0.016^{\mathrm{s}} \\ \text { Median } & 16 & \\ \text { Range (Min -max) } & (10-30) & \\ \text { ANC } & & \\ \text { Mean } \pm \text { SD } & 23.3 \pm 5.7 & \\ \text { Median } & 23 & \\ \text { Range (Min -max) } & (10-32) & \\ \text { Platelet } & & \\ \text { Mean } \pm \text { SD } & 24.1 \pm 6.5 & \\ \text { Median } & 24 & \\ \text { Range (Min -max) } & (16-32) & \end{array}$

Table II

Comparison of RET\%, Abs Ret and HFR in days $(n=50)$

$\begin{array}{lcc}\text { Ret\% } & & \\ \text { Mean } \pm \text { SD } & 18.5 \pm 6.8 & 0.013^{\mathrm{s}} \\ \text { Median } & 20 & \\ \text { Range (Min -max) } & (18-26) & \\ \text { Ret abs } & & \\ \text { Mean } \pm \text { SD } & 18.3 \pm 4.7 & 0.001^{\mathrm{s}} \\ \text { Median } & 18 & \\ \text { Range (Min -max) } & (16-32) & \\ \text { HFR } & & 0.450 \mathrm{n}^{\mathrm{s}} \\ \text { Mean } \pm \text { SD } & 20.3 \pm 6.8 & \\ \text { Median } & 18 & \\ \text { Range (Min -max) } & (10-32) & \end{array}$

\section{Discussion}

Limited studies were performed to evaluate utility of using flowcytometric analysis of reiculocytes to predict the bone marrow recovery after chemotherapy. Though the high cost of using flowcytometry or third generation automated blood cell counters for automated reticulocyte counts are presently a limiting factor in developing countries such as Bangladesh. But despite the fact, IRF can effectively serve as an additional index to indicate bone marrow recovery other than ANC. Dekoninck et al $(2002)^{15}$ found ret $\%$ recovery from 5 to 33 days (median 19), which was 5.5 days lathan for IRF. But this study found recovery in 20 days. Kuse et al. $(1996)^{16}$ confirmed that ARC recovered at a median of 10 days in acute leukaemia. But in this study ARC recovery occurred in 18 days. This study found IRF recovery at a median of 16 days but Das et al. $(2006)^{13}$ showed earlier IRF recovery at a median 21 days. Our study found recovery time for HFR at a median of 18 days. Riley $S(2001)^{5}$ found HFR recovery occurred from 4 to 33 days (median 18 day).

Our study found platelet recovery at a median of 24 days but Bhatnagar et al. $(2002)^{14}$ showed platelet count $\left(>100 \times 10^{9} /\right)$ recovered 4 days, prior to recovery of ANC above $0.5 \times 10^{9} / 1$ of all the parameters. This study found ANC recovery at a median of 23 days but Das et al. (2006) ${ }^{13}$ found ANC recovery between 10-35 days (median day 19).

This study concluded that the IRF parameter showed earlier haematopoietic recovery than the current practice of ANC recovery for monitoring in children with acute lymphoblastic leukemia after chemotherapy. This early laboratory indicator will guide the clinicians to make important therapeutic decisions, which will be economic savings and as well as live saving. Now a days, IRF is offered most of the third generation haematology analyzer. Moreover, this test is simple, quick, cost effective, reproducible and reliable tool on the automated haematology analyzer. Thus its potential use as a routine test to see the bone marrow recovery is important.

\section{Acknowledgement}

I would like to thank all the consultants, doctors and staff of the Department of Clinical Pathology, BSMMU, Dhaka for their sincere help in conducting this study. 


\section{References}

1. Baruchel A, Leblanc T, Schaison G, 1999, 'Pathology of acute lymphoblastic leukaemias', In: Lilleyman, Hann I, and Blanchette V (eds), Pediatric Haematology $2^{\text {nd }}$ ed, London, Harcourt Brace and Company, pp. 519-536.

2. Satake N, Yoon JM, 2009, 'Acute Lymphoblastic Leukaemia', E medicine Pediatric: medscape.com. 12:1-26

3. Kuse R, 1993, 'The appearance of reticulocytes with medium or high RNA content is a sensitive indicator of beginning granulocyte recovery after aplasiogenic cytostatic drug therapy in patients with $A M L^{\prime}$, Ann Hematol, 66: 213-214.

4. Greinix HT, Linkesch W, Keil F, Kalhs P, Schwarzinger I, Schneider B, Oesterreicher C, Brugger S, Kapiotis S, Lechner K Kalhs P, Schwarzinger I, Schneider B, Oesterreicher C, Brugger S, Kapiotis S, Lechner K, 1994, 'Early detection of hematopoietic engraftment after bone marrow and peripheral blood stem cell transplantation by highly fluorescent reticulocyte counts', Bone Marrow Transplant, 14: 307-313.

5. Riley S, Jonathon M, Ezra B,Goel R, Tidwell A, 2001, 'Reticulocytes and Reticulocyte Enumeration', Journal of Clinical Laboratory Analysis, 15:267-294.

6. Hoewen B, 1992, 'Reticulocyte maturation', Blood cells, 18:167-186.

7. Davis BH, Bigelow NC, 1989, 'Flow cytometric reticulocyte quantification using thiazole orange provides clinically useful reticulocyte maturity index', Arch Pathol Lab Med, 113: 684-689.

8. Remacha, 1994, 'Flow cytometric reticulocyte quantification in the evaluation of hematologic recovery. Spanish Multicentric Study Group for Hematopoietic Recovery', Eur J Haematol, 53: 293-297.

9. Hellgriel $K, 1993$, 'The clinical significance of reticulocyte determination', In: Basting T, $5^{\text {th }}$ eds. The Emerging Importance of Accurate Reticulocyte Counting. Miles, Inc. Diagnostic Division, Tarrytown: 7-10.
10. Davis BH, 1996, 'Immature reticulocyte fraction (IFR): by any name, a useful clinical parameter of erythropoietic activity', Lab Hematol, 2:2-8.

11. Bain BJ, Lewis SM, Bates I, 2008, 'Basic haematological techniques', In: Lewis SM, Bain B Bates I, Dacie and Lewis Practical Haematology, $10^{\text {th }}$ eds. Philadelphia: Churchill living stone, pp.25-57.

12 Imazu M, 2002, 'General Description of the Automated Hematology Analyzer, XT-2000i', Sysmex J Int, 12: 13-17.

13 Das R, Dip NB, Garewal G, Marwaha RK, Vohra H, 2006, 'Automated Reticulocyte response is a good predictor of bone-marrow recovery in pediatric malignancies', Pediatric Hematology and Oncology, 23:299-305.

14. Bhatnagor S, Chandra J, Naayanb S, 2002, 'Hematological Changes and Predičtors of Bone Marrow Recovery in Patients with Neutropenic Episodes in Acute Lymphoblastic Leukemia', Journal of Tropical Pediatrics, 48(4): 200-203

15. Dekoninck A, Brusselmans C, Goossens W, 2002, 'Indicators for hematopoietic recovery in patients after bone marrow transplantation or intensive chemotherapy' Department of laboratory Medicine, University Hospital Leuven, Leuven, Belgium, pp.39.

16. Kuse $R$, Foures $C$, Jou JM, d'Onofrio G, Paterakis $G$, 1996, 'Automated reticulocyte counting for monitoring patients on chemotherapy for acute leukaemias and malignant lymphomas', Clin Lab Haematol , 18(1): 39-43. 